\title{
Hearing Loss-a Camouflaged Manifestation of COVID 19 Infection
}

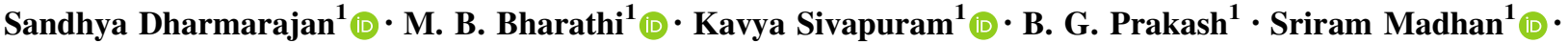 \\ Ashima Madhu ${ }^{1}$ G. Nivetha Devi ${ }^{1}$ Shaik Ayesha Aliya ${ }^{1}$ S. B. Ramya ${ }^{1}$
}

Received: 6 February 2021 / Accepted: 17 April 2021/Published online: 10 May 2021

(C) Association of Otolaryngologists of India 2021

\begin{abstract}
The symptomatology of novel Severe Acute Respiratory Syndrome Corona virus type 2 infection runs the entire gamut of mild to moderate and serious illness among the affected individuals. As listed in recent literature, respiratory, cardiovascular, gastrointestinal, olfactory and gustatory systems are commonly involved. With the growing knowledge about the disease, varied manifestations have been identified and lately, otorhinolaryngology dysfunctions in COVID 19 have been described. Hearing loss in COVID era is one of the emerging areas of concern and calls for further research in the field for the better understanding and treatment of this entity. This study was designed to assess the audiological profile among 100 mild to moderately affected COVID-19 individuals, so as to make a contribution to the emerging literature on otologic manifestations in COVID 19. In our case series, high frequency hearing loss and referred OAE was noted among significant number of COVID 19 positive patients. This was even observed in patients without any otologic symptoms. Hence, early identification and intervention if required helps to give a better quality of life to the patient.
\end{abstract}

Keywords Hearing loss · COVID 19 .

Pure tone audiometry · Oto acoustic emissions

M. B. Bharathi

mbbharathi@jssuni.edu.in

1 Department of ENT, Head and Neck Surgery, JSS Medical College, JSS Academy of Higher Education and Research (JSSAHER), Mysuru, Karnataka 570004, India

\section{Introduction}

The outbreak of novel Severe Acute Respiratory Syndrome Corona virus type 2 (nSARS-CoV-2) infection in Wuhan, China in December 2019 has had a catastrophic impact on the world, resulting in over 2.2 million deaths globally $[1,2]$. The world envisioned sufferings of unprecedented proportions as mankind endured losses in terms of health, finances and importantly, life. The symptomatology of COVID 19 runs the entire gamut of mild to moderate and serious illness among the affected individuals. As listed in recent literature, it commonly involves respiratory, cardiovascular, gastrointestinal, olfactory and gustatory systems [3-5]. With the growing knowledge about the disease, varied manifestations have been identified and lately, otologic dysfunctions in COVID 19 have been described [6].

Viral infections have been implicated in causing hearing loss, the reasons being direct or indirect inner ear damage [7-9]. Hence, the prospects of involvement of the auditory system following COVID 19 has been under scrutiny, the frequently reported otologic symptoms being tinnitus and hearing loss $[6,10]$. Sensorineural hearing loss has been more commonly implicated in these patients although a few have exhibited conductive hearing loss as well $[6,10-14]$. Various theories explaining the involvement of the ear following SARS CoV-2 infection have been postulated, which include immune mediated damage, haematogenous spread, ischaemia theory, inflammation of auditory pathway components, presence of angiotensin converting enzyme receptors (ACE2) receptors in neurons and glial cells [6-9].

Hearing loss in COVID era is one of the emerging areas of concern and calls for further research in the field for the better understanding of this entity. This study was designed to assess the audiological profile among 100 mild to 
moderately affected COVID-19 individuals, so as to make a contribution to the emerging literature on otologic manifestations in COVID 19.

\section{Materials and Methods}

\section{Study Design}

A cross sectional observational study of audiological assessment was done among the patients admitted with SARS COV 2 infection in a tertiary care hospital designated for COVID 19 patients. This study was carried out over a period of 3 months from September 15th 2020 to December 15th 2020.

\section{Study Population}

This study included 100 patients admitted in our hospital COVID 19 wards who met the inclusion criteria as follows:

(1) Age 21-60 years

(2) COVID 19 RTPCR Positive

(3) No prior history of any ear symptoms (hard of hearing, ear discharge, tinnitus, ear pain, giddiness)

Exclusion criteria:

(1) Age $>60$ years

(2) History of previous ear symptoms, tympanic membrane perforations or ear surgeries

(3) Patients with history of Diabetes mellitus, Renal disease, patients on ototoxic drugs.

(4) Patients not maintaining saturation and requiring $\mathrm{O} 2$ support

(5) Intensive care unit patients

\section{Methodology}

Patients admitted to our Hospital COVID wards with mild to moderate symptoms were enrolled in the study after obtaining prior consent from them. The particulars pertaining to each patient were compiled through a structured proforma (Table 1). After a thorough history taking as well as clinical examination which included otoscopic examination of the ears, these patients were then subjected to audiological evaluations by Pure Tone Audiometry and Oto Acoustic Emissions in a sound proof room. Pure tone thresholds were measured in both ears at 500, 1000, 2000, 4000 and $8000 \mathrm{~Hz}$. Transient Evoked Oto Acoustic Emissions (TEOAE) were measured in all the patients and the results obtained were documented.

\section{Results}

A total of 100 patients were included in the study. Among these 58 were male patients and 42 were female patients. There were 23 patients in the age group of 20-30 years, 31 in $31-40$ years and 28 patients in $41-50$ years age and 18 patients between 50 and 60 years. Of the 100 patients, 27 patients had hypertension, 6 patients had history of ischemic heart disease. Only one patient had history of using propranolol. 22 patients in total received remedesivir injections for their symptoms.

31 among the 100 patients had ear symptoms, the most common being tinnitus (39\%) and the least complained symptom as giddiness (10\%) (Fig. 1).

Otoscopic examination showed normal external auditory canal and tympanic membrane in 95 patients, 4 patients had retracted tympanic membrane and 2 had dull TM.

Pure tone audiometry measured in both ears at 500, 1000, 2000, 4000 and $8000 \mathrm{~Hz}$ revealed high frequency hearing loss among these patients and the most common pattern of hearing loss was sensorineural hearing loss(SNHL). 6 patients had conductive hearing loss(CHL) (Fig. 2).

Sensorineural hearing loss was present in 53 patients in total. Among 53 patients, 11 patients had symptoms of hearing loss whereas rest 42 patients did not report hard of hearing as a symptom. Sensorineural hearing loss was present in all the individuals who received remdesivir in their treatment. Age wise distribution of patients and sensorineural hearing loss is shown in Fig. 3a, b respectively.

Among the 100 patients, a total of 49 patients had a referred $\mathrm{OAE}$ in both the ears, with majority of the patients having high frequency hearing loss.

Among the 31 patients who complained of ear symptoms, 18 had a referred OAE, so did 31 among the 69 patients who never had any ear symptoms (Fig. 4).

\section{Discussion}

Growing evidence among the COVID 19 positive subjects mandates the hearing assessment in subjects tested positive for the virus. Early identification of hearing loss in these individuals would have a far-reaching impact when looking at the quality of life post COVID, as it is often a much neglected symptom. In our case series, hearing loss was noted in $11 \%$ of the patients who had symptom of hearing loss and $48 \%$ of the patients who did not have any symptom of hearing loss (42\%-SNHL, $6 \%-\mathrm{CHL})$. Sriwijitalai et al., reported the first case of SNHL in a COVID 19 positive patient in april 2020 [11]. Following this few other reports also have been published relating SNHL in COVID 19 positive patients [10, 12-14]. 
Table 1 Proforma

\begin{tabular}{lll}
\hline S.No & Domains & Details \\
\hline 1 & Name & \\
2 & Age/Sex & \\
3 & Contact number & \\
4 & Tested positive on & \\
5 & Date of admission & \\
6 & Date of evaluation & \\
7 & Ear complaints & Ear pain/discharge, hard of hearing, tinnitus, aural fullness, giddiness \\
8 & Pre-existing ear conditions & Ear pain/discharge, hard of hearing, tinnitus, aural fullness, giddiness \\
9 & New onset of ear symptoms & Type 2 DM, HTN, Renal disease, Cardiovascular diseases \\
10 & Co-morbidities & Furosemide/ Amikacin/ Streptomycin/ Propranolol/ Indomethacin \\
11 & Drug history & Yes/No \\
12 & Use of Remdesivir &
\end{tabular}

Ear-Right Left

External auditory canal-

Tympanic membrane-

Pure tone audiometry: Average thresholds Type of hearing loss

Right-

Left-

OAE-Pass/Referred

If referred-High frequency/ low frequency

Fig. 1 Most common ear symptoms



Hearing loss could be viral induced, immune complex mediated, vascular occlusion or cellular stress response [7, 8]. Literature shows various reports of viral mediated hearing loss most commonly implicated viruses being herpes virus and cytomegalovirus [6, 12, 15, 16]. SARS $\mathrm{COV} 2$ virus is also thought to be responsible in causing sensorineural hearing loss through inflammatory response on cochlear hair cells. Presence of ACE2 receptors in brain, medulla oblongata, temporal lobe has facilitated the entry of SARS CoV2 affecting the brainstem and hearing centres leading to inflammatory response through cytokine release. This could lead to neurologic and otologic manifestations in COVID 19 affected patients [17]. Samir et al., reported a case report of SSNHL in a patient who presented with complaints of sudden onset of left side hearing loss and gradually worsening tinnitus on day 3 of COVID 19 infection. Patient was managed with 3 doses of intratympanic methyl prednisolone and repeat audiogram after recovery showed significant improvement in hearing loss [13]. 


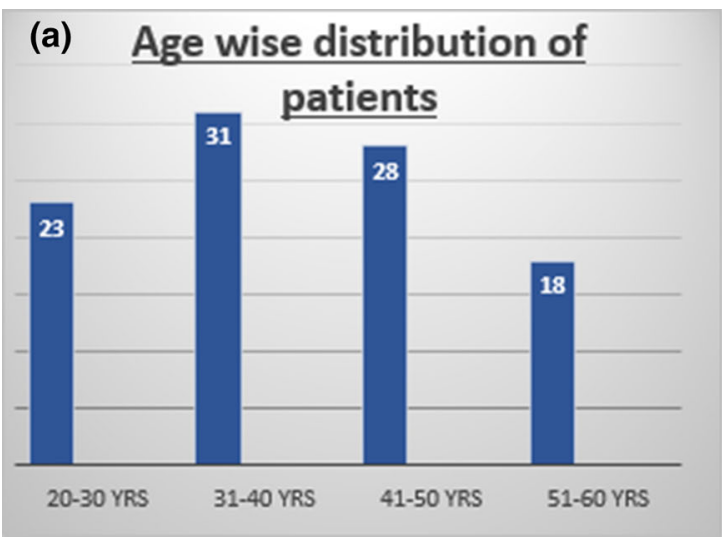

Fig. 2 Pure tone average thresholds

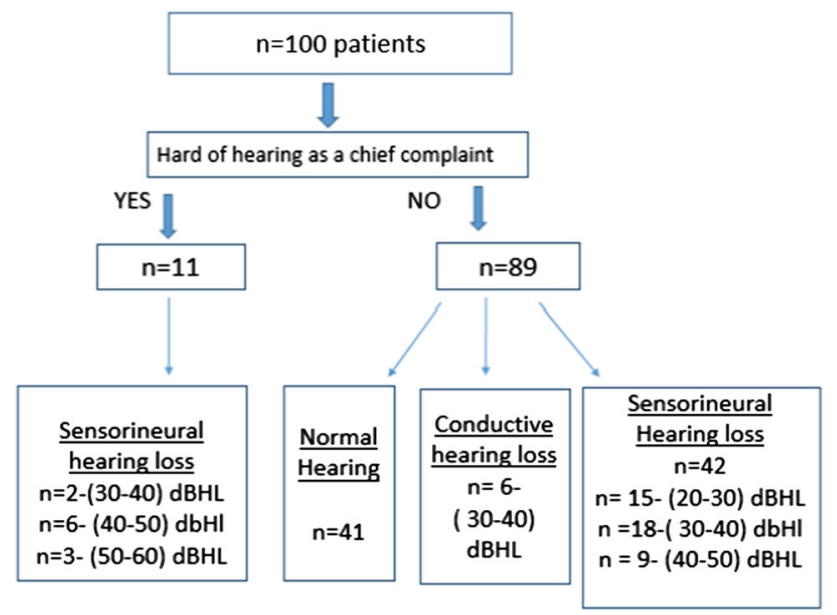

Fig. 3 Age wise distribution of patients and sensorineural hearing loss

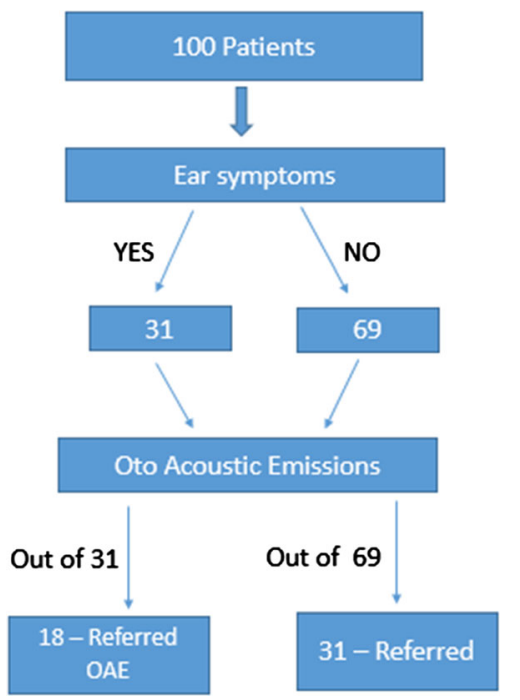

Fig. 4 Transient Evoked Oto Acoustic Emissions (TEOAE)

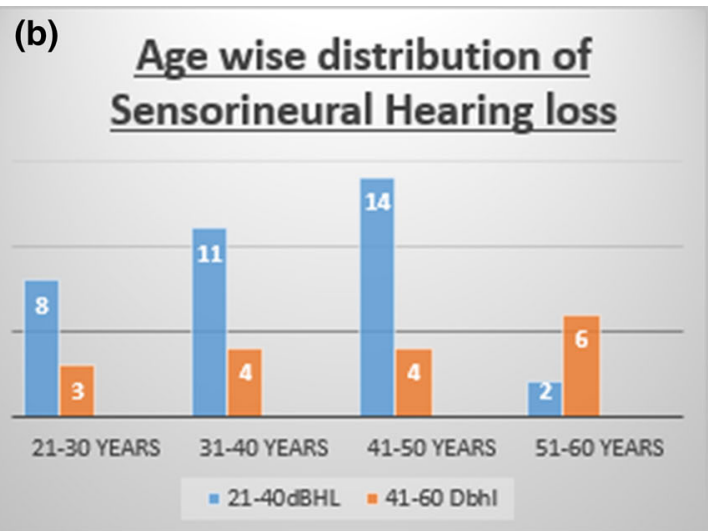

Chantal et al., reported a case of 60 year old male patient, a confirmed case of COVID 19 infection with severe symptoms under ICU care for 13 days complained of hearing loss and tinnitus. Audiological evaluation showed complete deafness on right side and profound sensorineural hearing loss on left side and MRI brain revealed pronounced contrast enhancement in right cochlea, decreased fluid signal in basal turn showing an inflammatory process in cochlea. Patient was managed by cochlear implantation in right ear and intratympanic triamcinolone in the left ear [14]. This study showed the importance of audiological evalution and radio-imaging as and when required when COVID 19 positive patients present with severe hearing loss and emphasized the need for prompt management for better quality of life of the patient.

Recent systematic review of 5 case reports and 2 case series by Maharaj et al., on otological dysfunction in COVID 19 patients showed hearing loss as the most common presenting symptoms followed by tinnitus, vertigo and otalgia. They concluded that there is significant association of otological disorers, hearing loss in specific in COVID-19 patients [6]. Hearing loss can be part of the clinical spectrum of COVID-19 and may in some cases signal the onset of the disease.

A comparative study of audiological evaluation between 20 asymptomatic COVID-19 positive patients and 20 normal hearing patients by Mustafa et al., revealed significant high frequency pure tone thresholds hearing loss and referred TEOAE in asymptomatic COVID 19 patients indicating inimical effects of COVID 19 infection on cochlear hair cell functions [12]. In our study, high frequency pure tone threshold sensorineural hearing loss and referred TEOAE was noted even in many patients without symptoms of hearing loss. 6 of our patients had conductive hearing loss probably due to the presence of middle ear effusion caused by the infection in nasopharynx. 
This shows the possibility that SARS CoV2 infection has detrimental effects on cochlear function. Hearing assessment through audiometry in patients with COVID19 infection will aid us in early identification of any otological adverse effects. As per this case series, it can be illustrated that mere absence of symptoms does not rule out auditory involvement and hence early intervention will help in improving the quality of life of the patient.

Our case series includes 100 patients which is a high number among the publications till date. High frequency hearing loss and referred OAE was noted among significant number of COVID 19 positive patients. All the patients received intravenous steroids as per the institution COVID 19 treatment protocol. So far, new onset hearing loss has been documented in COVID 19 positive individuals. But whether this hearing loss is transient or permanent is yet to be established. We present this case series as an initial work up. These patients will be followed up at regular intervals of 3 and 6 months for repeat audiometric evaluation. A detailed remark can be made after a follow up period of 6-9 months.

\section{Conclusion}

Otologic disorders among COVID19 affected individuals is becoming increasingly evident day by day. Assessment of hearing loss is of utmost importance to safeguard the cochlear function and prevent the progressive deterioration of cochlear hair cells $[13,14]$. High frequency hearing loss and referred OAE was noted among significant number of COVID 19 positive patients in our case series. Early identification and intervention will help to give a better quality of life to the patient. This case series is an initial work up on COVID 19 positive individuals. A detailed review of whether this hearing loss is transient or permanent in nature can be specified only after regular follow up of these individuals.

Acknowledgement We thank all the post graduates of Department of ENT, Head and Neck Surgery of our institute for their extra efforts by wearing personal protective equipment and performing audiometry among COVID 19 positive patients

Funding No external funding has been received.

Data Availability Data transparency has been maintained.

\section{Declarations}

Conflict of interest The authors declare that they have no conflict of interest.
Ethical Approval Appropriate ethical clearance has been obtained from the institute.

Human or Animal Rights It was an observational study done on the human participants after obtaining the informed consent from all the individuals.

Consent for Publication Appropriate consent for publication taken.

Informed Consent Informed consent was obtained from all individual participants included in this study.

\section{References}

1. COVID-19 Worldwide Dashboard - WHO Live World Statistics

2. (COVID-19) virus - Coronavirus disease 2019 (COVID-19)

3. Kordzadeh-Kermani E, Khalili H, Karimzadeh I (2020) Pathogenesis, clinical manifestations and complications of coronavirus disease 2019 (COVID-19). Future Microbiol 15(13):1287-1305

4. Sobh E, Abuarrah E, Abdelsalam KG, Awad SS, Badawy MA, Fathelbab MA, Aboulfotouh MA, Awadallah MF (2020) Novel coronavirus disease 2019 (COVID-19) non-respiratory involvement. Egypt J Bronchol 14(1):1-6

5. Tong JY, Wong A, Zhu D, Fastenberg JH, Tham T (2020) The prevalence of olfactory and gustatory dysfunction in COVID-19 patients: a systematic review and meta-analysis. OtolaryngolHead Neck Surg 163(1):3-11

6. Maharaj S, Bello Alvarez M, Mungul S, Hari K (2020) Otologic dysfunction in patients with COVID-19: a systematic review. Laryngoscope Investig Otolaryngol 5(6):1192-1196

7. Chen X, Fu YY, Zhang TY (2019) Role of viral infection in sudden hearing loss. J Int Med Res 47(7):2865-2872

8. Saniasiaya J (2020) Hearing loss in SARS-CoV-2: What do we know? Ear Nose Throat J 1:0145561320946902

9. Cure E, Cure MC (2020) Comment on "Hearing loss and COVID-19: A note". Am J Otolaryngol 41(4):102513

10. Koumpa FS, Forde CT, Manjaly JG (2020) Sudden irreversible hearing loss post COVID-19. BMJ Case Rep CP 13(11):e238419

11. Sriwijitalai W, Wiwanitkit V (2020) Hearing loss and COVID19: a note. Am J Otolaryngol 41(3):102473

12. Mustafa MW (2020) Audiological profile of asymptomatic Covid-19 PCR-positive cases. Am J Otolaryngol 10:102483

13. Rhman SS, Wahid AA (2020) COVID-19 and sudden sensorineural hearing loss, a case report. Otolaryngology Case Reports. https://doi.org/10.1016/j.xocr.2020.100198

14. Degen C, Lenarz T, Willenborg K (2020) Acute profound sensorineural hearing loss after COVID-19 pneumonia. Mayo Clin Proc 95(8):1801-1803

15. Taneja MK (2020) Deafness in COVID-19. Indian J Otol 26(2):68

16. Kalcioglu MT, Cag Y, Kilic O, Tuysuz O (2020) May COVID-19 cause sudden sensorineural hearing loss? Int J Infect Dis.

17. Helms J, Kremer S, Merdji H, Clere-Jehl R, Schenck M, Kummerlen C, Collange O, Boulay C, Fafi-Kremer S, Ohana M, Anheim M, Meziani F (2020) Neurologic features in severe SARS-CoV-2 infection. N Engl J Med 382(23):2268-2270. https://doi.org/10.1056/NEJMc2008597

Publisher's Note Springer Nature remains neutral with regard to jurisdictional claims in published maps and institutional affiliations. 